\title{
MEDICINE
}

\section{THE STUDY OF CELLULAR FACTORS OF IMMUNITY IN PATIENTS WITH NON-HODGKIN'S LYMPHOMAS}

\author{
Abdiganiyeva S. R., Tillyashaykhov M. N., Abdurakhmonov D. A., Tuidzhanova Kh. Kh. \\ Republican specialized scientific-practical medical center of oncology and radiology of Ministry of \\ health of the Republic of Uzbekistan
}

DOI: https://doi.org/10.31435/rsglobal_sr/31072019/6608

\section{ARTICLE INFO}

Received 24 May 2019

Accepted 10 July 2019

Published 31 July 2019

\section{KEYWORDS}

adaptive cellular immunity, humoral immunity, polychemotherapy, lymphomas, non-Hodgkin lymphoma.

\begin{abstract}
The purpose of this study was to study the basic cellular indicators of immunity in patients with non-Hodgkin's lymphomas to substantiate the immunopathogenetic features of the course of the disease. It turned out to be important that patients were divided into 2 groups with virus carrier and without virus carrier.

An analysis of the results allowed us to identify pronounced changes in the cellular immunity that manifested themselves by suppressing the expression of $\mathrm{CD} 3+, \mathrm{CD} 3+\mathrm{CD} 4+$, IRI, against this background, an increase in the expression of CD3 + CD8 +, CD16 + and CD20 + B cells, as well as increased expression of activation molecular markers of CD38 + lymphocytes, CD95 +. Deep T-cell immunodeficiency was detected against the background of pathological activation of lymphocytes, which is clinically often reflected by frequent relapses, an unfavorable course of the disease, and treatment results.
\end{abstract}

Citation: Abdiganiyeva S. R., Tillyashaykhov M. N., Abdurakhmonov D. A., Tuidzhanova Kh. Kh. (2019) The Study of Cellular Factors of Immunity in Patients with Non-Hodgkin's Lymphomas. Science Review. 6(23). doi: 10.31435/rsglobal_sr/31072019/6608

Copyright: $\quad$ C 2019 Abdiganiyeva S. R., Tillyashaykhov M. N., Abdurakhmonov D. A.,
Tuidzhanova Kh. Kh. This is an open-access article distributed under the terms of the Creative Commons
Attribution License (CC BY). The use, distribution or reproduction in other forums is permitted, provided the
original author(s) or licensor are credited and that the original publication in this journal is cited, in accordance with
accepted academic practice. No use, distribution or reproduction is permitted which does not comply with these terms.

Non-Hodgkin's lymphomas (NHL) - a heterogeneous group of tumors of the blood system, characterized by various clinical course, localization, morphological, immunological; cytogenetic features, and results of therapy $[12,16]$. In recent years, the incidence of NHL has increased $[6,10,13,15]$. Men get sick about 3 times more often than women $[1,2,4,7,9,9,14]$.

Cells of various morphoimmunological characteristics are the substrate of NHL, modern ideas about which allow us to rethink the basics of isolation! and the systematization of various options for NHL $[1,5,7,8]$. The last years of the development of oncohematology were marked by the emergence of new research methods: immunophenotyping, immunohistochemistry, molecular genetics, which along with morphological studies contributed to a deeper understanding of the pathogenetic mechanisms of the formation of NHL. This is reflected in modern classifications of lymphomas (classification of the EuropeanAmerican group for the study of lymphomas 1994., classification of tumors of the hematopoietic and lymphoid tissues of the WHO, 2001, 2008). The use of immunodiagnostics methods led to the isolation of T-, B- and cellular NHL, and within each group - several options (from immunologically immature and mature cells) $[9,11,12,16]$. Later, taking into account the expression of certain clusters of cell differentiation, some variants were transformed into separate nosological units: primary mediastinal (thymic) B-large cell lymphoma (BLCL), anaplastic large-cell lymphoma (LCL) positive and negative [1,2,4,7, 9.14]. In childhood, lymphoblastic lymphomas from progenitor cells (LBL) are most often diagnosed, and from mature cell NHL, Burkitt's lymphoma (BL), LCL characteristics of the tumor, in particular, the group. Only an integrated approach that takes into account the peculiarities of the morphological picture and uses an 
expanded diagnostic immunological panel of antibodies, which is aimed at identifying not only the markers of mature $\mathrm{T}$ and $\mathrm{B}$ cells, but also the antigens of progenitor cells, makes it possible to correctly diagnose $[4,5,7,10]$. The main treatments for NHLremain polychemotherapy and radiation therapy. Isolation of new nosological forms in NHL group led to the emergence of various PCT programs followed by their gradual intensification (COP, COMP, LMB-81.84.86.89, NHL-BFM90 / 95) [12.16].

Based on the immunological characteristics of tumor cells, targeted drugs - monoclonal antibodies (anti-C020 - rituximab, zevalin, anti-CD22 - epratuzumab) have now begun to be used for therapeutic purposes. As a result of the use of modern therapy programs, the indicators of the overall, event-free and relapse-free survival of individuals with NHL have improved, reaching by now $85-91 \%$ [2.6]. The use of targeted therapy has improved B-NHL treatment outcomes. However, the problem of improving treatment, which directly depends on the state of the immune system, the presence and degree of secondary immunodeficiency, and the individual development of the disease, remains urgent.

At present, great attention is paid to the study of immunological mechanisms in the development and progression of lymphomas. An important issue remains the immunodiagnostics of cellular and humoral immunity. As the literature shows, publications devoted to the study of immunity are sporadic. Basically, articles are devoted to clinical study and the description of the results of treatment methods, while there are no works of a pathogenetic nature. Reliable data can only be obtained by analyzing a sufficiently large amount of clinical material, which makes it possible to study the frequency of occurrence of various NHL variants; comparison of clinical, morphological and immunological data, as well as treatment results for T- and B-cell lymphomas, which opens up the possibility of improving the diagnosis and individualization of chemotherapy programs depending on the immunophenotypic characteristics of tumor cells.

The aim of the study. To study the basic cellular indicators of immunity in patients with nonHodgkin's lymphomas to substantiate the immunopathogenetic features of the course of the disease.

Materials and research methods. In our study, patients with non-Hodgkin's lymphomas (NHL) were divided into 2 groups: group I - patients with established virus carriers; group II - patients in the control group with no virus carrier. In the distribution of NHL patients by age, it was found that virus carriage leads to a rejuvenation of the disease: the peak incidence rate in group I is up to 30 years, while the same indicator for group II is over 50 years. The results of the analysis are presented in table 1.

Table 1. Distribution of patients by age and gender

\begin{tabular}{|c|c|c|c|c|c|c|c|c|}
\hline \multirow{3}{*}{ Age, years } & \multicolumn{4}{|c|}{$\begin{array}{c}\text { group I } \\
\text { (virus carrier), } n=23\end{array}$} & \multicolumn{4}{|c|}{$\begin{array}{c}\text { group II } \\
\text { (control), } \mathrm{n}=18\end{array}$} \\
\hline & \multicolumn{2}{|c|}{ Men } & \multicolumn{2}{|c|}{ Women } & \multicolumn{2}{|r|}{ Men } & \multicolumn{2}{|c|}{ Women } \\
\hline & Abs & $\%$ & Abs & $\%$ & Abs & $\%$ & Abs & $\%$ \\
\hline$<30$ & 11 & $47,8 \pm 7,8$ & 4 & $17,3 \pm 5,9$ & - & & 2 & $11,1 \pm 4,9$ \\
\hline $31-40$ & 1 & $4,3 \pm 2,2$ & - & & 1 & $5,5 \pm 2,7$ & 1 & $5,5 \pm 2,7$ \\
\hline $41-50$ & 2 & $8,6 \pm 4,4$ & 1 & $4,3 \pm 2,2$ & - & & 1 & $5,5 \pm 2,7$ \\
\hline $51-60$ & 1 & $4,3 \pm 2,2$ & - & & 5 & $27,7 \pm 6,9$ & 3 & $16,6 \pm 5,8$ \\
\hline $61-70$ & 2 & $8,6 \pm 4,4$ & - & & 1 & $5,5 \pm 2,7$ & - & \\
\hline$>70$ & 1 & $4,3 \pm 2,2$ & - & & 3 & $16,6 \pm 5,8$ & 1 & $5,5 \pm 2,7$ \\
\hline
\end{tabular}

The age of sick men with virus-bearing was $35.6 \pm 4.56$ years, among women $-27.2 \pm 5.24$ years, in the control group, the average age of men was $60.7 \pm 3.80$ years, women $-45.5 \pm 6,97$ years old. The proportion of women in group I was $21.7 \pm 6.44 \%$, men $-78.3 \pm 6.44 \%$; in group II, women accounted for $44.4 \pm 7.75 \%$, men - $55.6 \pm 7.75 \%$.

In all patients, the diagnosis was established on the basis of data and the results of a comprehensive study (clinical, biochemical, radiological, ultrasound, CT, myelogram, morphological).

The lesion areas for non-Hodgkin's lymphomas in patients with viral infection (group I) most often occurred in the cervical and axillary $1 /$ nodes (more than 50\%), then in the mediastinal, supraclavicular, retroperitoneal and inguinal (25-45\%), the lesion was least noted. iliac $1 /$ nodes, spleen, Valdeyer ( $20 \%$ or less).

In control group II, the picture of lymph node lesions differed from the experimental group patients with affected cervical 1 / nodes were more than $70 \%$, while the remaining lesion zones were less common: axillary, supraclavicular, inguinal 1 / nodes malignantly transformed in 30-40\% of cases; mediastinal, retroperitoneal, iliac - in less than $20 \%$ of cases; Valdeyer ring damage was observed in more than $60 \%$ of patients. 
Thus, viral infection changes the picture of lymph node damage in patients with NHL in comparison with patients without viral load: in group I, axillary, mediastinal, supraclavicular, retroperitoneal, and retroperitoneal / inguinal 1 / nodes were transformed malignantly (25-45\%). Group I patients were most often infected with HSV herpes simplex virus and Epstein-Barr virus $(65-80 \%)$. Cytomegalovirus infection (CMV), human papilloma virus (HPV) in $43.4 \pm 7.7 \%$ of patients, and Varicella-Zoster virus were found in $26.0 \pm 6.8 \%$ and $8.6 \pm 4.4 \%$, respectively.

The examination included patients undergoing examination in the chemotherapy department. All patients underwent clinical and laboratory blood tests, which included the study of a general analysis of blood and urine, biochemical and immunological parameters, as well as the blood coagulation system. Patients underwent a comprehensive clinical and instrumental examination aimed at clarifying the prevalence of the tumor process and identifying existing complications.

Immunological studies included the study of cellular and humoral parameters of the immune system of patients with OC. Determination of cellular immunity $(\mathrm{CD} 3+, \mathrm{CD} 3+\mathrm{CD} 4+, \mathrm{CD} 3+\mathrm{CD} 8+$, $\mathrm{CD} 16+, \mathrm{CD} 20+$ ), as well as identification of activation markers of lymphocytes (CD25 +, CD38 + and CD95 +) was carried out by flow cytometry using Accuri C6 (USA) using monoclonal antibodies.

When conducting a statistical analysis of the data presented in the work, the results of the study were entered into databases prepared in the Microsoft Excel XP program. Numerical (continuous) values were presented as arithmetic means and mean errors $(\mathrm{M} \pm \mathrm{m})$. Comparison of quantitative characteristics was carried out using Student's criterion, for continuous variables - paired Student's criterion. $\mathrm{P}<0.05$ was taken as a boundary comparative criterion of statistical significance.

The obtained research results and their discussion. Thanks to the modern achievements of fundamental immunology, molecular biology, new biologically significant indicators have now appeared in the arsenal of researchers, which can help the practitioner, in particular in the diagnosis, treatment and prognosis of diseases, as well as in the choice of immunotropic therapy. In this regard, in recent years, much attention has been paid to immunological markers that can help in identifying an immunodeficiency state, diagnosing, predicting and preventing relapse of the disease, which is a kind of indicator of the nature of the course of the disease, especially in cancer [11,15].

The main factors of immunity that have been studied and will be presented below include cellular factors of immunity, such as population and subpopulation markers of lymphocytes. It should be noted that the listed immunity parameters are specific factors, but studying them against a specific nosology and comparing the results with the clinical manifestations of the disease are definitely important, specific value, because it is the elements of the immune system that accompany all processes of pathogenesis, the development of the disease, its progression and outcome. It is known that all malignant processes are related to immunodeficiency conditions, accompanied by immunosuppression of any parts of the immune system [6,13]. Therefore, the study of the immunoreactivity status of cancer patients is an important factor necessary to establish the depth of immunodeficiency, to predict the disease and, most importantly, to identify the most radical methods of therapy, including immunotropic therapy in the future.

The immunological parameters of patients with NHL were analyzed before the start of complex treatment to identify pathogenetic characteristics of the response. Analysis of the results showed that the average content of leukocytes in peripheral blood in all groups of patients was reduced compared with the value of the control group.

Moreover, a significant decrease in the content of leukocytes was detected in groups of patients before treatment compared with data from a group of practically healthy individuals. The highest leukocyte count was detected in the group of patients and amounted to $6280.9 \pm 280.7 \mathrm{cl} / \mu 1$. The study of the relative content of the total pool of lymphocytes between the studied groups of patients showed that the number of lymphocytes was significantly suppressed in the group of patients compared with the value of the control group. It was found that the most significantly low value of lymphocytes was observed in groups of patients before treatment with virus carrier. So, the level of lymphocytes in the group of patients with virus carrier was $29.3 \pm 1.2 \%$, while in the control group it was $33.8 \pm 1.11 \%$.

It was found that phenotypic markers of lymphocytes include such markers as CD3 +, CD3 + CD4 ,$+ \mathrm{CD} 3+\mathrm{CD} 8+, \mathrm{CD} 20+$. It is widely shown in the literature that the triggering and regulation of the effectiveness of the immune response is largely determined by the specific antigen of T-lymphocytes. Responsible for this function are antigen-recognizing receptors - TCR. It is known that the degree of surface expression of $\mathrm{CD} 3$ + receptors on the membrane of T-lymphocytes reflects its transmissible function and allows to identify the total number of T-lymphocytes $[3,12]$. Thus, the analysis of the immunophenotype of CD3 + T-lymphocytes in patients with NHL showed that the presence of a reliable suppression of the 
expression of $\mathrm{CD} 3+$ on T-lymphocytes is observed in all groups of patients compared with data from a practically healthy group $(\mathrm{p}<0.05)$. The lowest CD3 + value was observed in the group of patients with virus carriers before chemotherapy. Obviously, a decrease in the total pool of T-lymphocytes $(\mathrm{CD} 3+)$ was observed mainly due to the suppression of the expression of CD3 + CD4 +. A study of the expression of $\mathrm{CD} 3+\mathrm{CD} 4+$ on T-lymphocytes, which are the main regulatory cells of the immune system, showed that the lowest value was noted in groups of patients before treatment $(p<0.05)$. The expression of CD3 $+\mathrm{CD} 4+$ was most suppressed in the group of patients with NHL with virus carrier. It has been shown in the literature that the CD4 + T-cell response to tumor proteins is an important cellular defense mechanism of the macroorganism, since CD4 + T-helpers stimulate antibody production by B-lymphocytes and activate CD8 + T-lymphocytes specific for tumor cells $[2,5,7,9,13]$. The analysis showed that in the group of patients with virus-bearing CD3 $+\mathrm{CD} 4+$ it was $21.4 \pm 1.3 \%$, while in the groups of patients without virus-bearing it was $25.8 \pm 0.8 \%$, and in the group of practically healthy individuals it was $35.7 \pm 1.14 \%$.

Cytotoxic CD3 + CD8 + T-lymphocytes play an important role in the pathogenesis of cancer $[4,8]$. It is proved that the function of these cells is the recognition of antigens on the cell surface in combination with MHC class 1 molecules. Since they are present on almost all nuclear cells of the body, any cell carrying MHC class 1 molecules in combination with an antigenic peptide can activate a clone of cytotoxic T lymphocytes. The biological role of this activation is the removal of mutant cells $[4,9]$. CD3 + CD8 + T-lymphocytes play a major role in the elimination of the virus, which is caused, on the one hand, by their ability to cause the death of infected cells expressing the corresponding peptides presented by MHC class 1 molecules, and, on the other hand, the ability to secrete antitumor factors - cytokines $[4,7,11,16]$.

Analysis of the expression of CD3 + CD8 + on T-lymphocytes revealed a significant increase in all groups of patients compared with the value of the control group. It should be noted that the maximum increase in CD3 + CD8 + was detected in the group of patients before treatment $(p<0.05)$. An analysis of the values of CD3 + CD8 + on T-lymphocytes between the studied groups of patients shows that before treatment, the expression of CD3 + CD8 + was significantly increased and amounted to $37.9 \pm$ $1.72 \%$. The immunoregulatory index (IRI), which is the ratio of the values of CD3 + CD4 + / CD3 + CD8 +, is of significant importance in secondary immunodeficiency states. It is known that in normal IRI in healthy individuals averages $1.62 \pm 0.02$. Obviously, suppression of the expression of CD3 + CD4 + against the background of increased expression of CD3 + CD8 + leads to a decrease in IRI. According to our data, the lowest decrease in IRI is observed in the group of patients before treatment with virusbearing compared with the data of patients without virus-bearing $(p<0.05)$. Therefore, pronounced immunosuppression was characteristic of patients with NHL in groups of patients before treatment with virus carrier. Apparently, a decrease in IRI is an important criterion for the depth of the T - cell immunodeficiency state, especially against the background of infection with latent chronic viruses.

Further, the expression of killer cells, which are the third population of lymphocytes providing maintenance of genetic homeostasis, which are phenotypically and functionally significantly different from $\mathrm{T}$ and $\mathrm{B}$ lymphocytes, was studied [5,8]. Killer lymphocytes belong to the category of the main effectors of natural or innate immunity, which are able to lyse target cells or carry out antibody-dependent cellular cytotoxicity. They perform the functions of the first line of defense before immune T-lymphocytes and specific antibodies arise [5,7]. Killer cells with CD16 + phenotypes were studied. A significant increase in the expression of CD16 + was revealed in all groups of patients. It was shown that the highest expression of $\mathrm{CD} 16+$ is observed in the group of patients with virus carrier $(\mathrm{p}<0.05)$. So, in the group of patients before treatment, the expression of CD16 + was $27.6 \pm 1.1 \%$, in the control group (without virus carrier) $-24.5 \pm$ $1.31 \%$, in the group of practically healthy individuals $-16.7 \pm 1.4 \%$. Consequently, the highest expression of CD16 + was observed in groups of patients prior to the start of chemotherapy with virus carrier.

A study of the expression of CD20 + on B-lymphocytes, which are the main regulatory cells of the immune system and are important in the development of humoral immunity, showed that the expression of CD20 + was significantly increased in all groups of patients $(p<0.05)$. Despite this, the study of the content of B-lymphocytes is an important criterion to assess the depth of immunodeficiency and determine the next steps in terms of diagnosis and treatment. Next, activation markers of peripheral blood lymphocytes were studied. It is known that the activation markers of lymphocytes began to be studied relatively recently, therefore, small works on the functional activity of activation markers of lymphocytes, in particular in oncological processes, have been reported in the literature [15,16]. From the available literature data, it is clear that the study of activation markers of lymphocytes, especially in proliferative processes, is of great scientific and practical importance. analysis of activation markers of lymphocytes allows one to study the processes of activation, proliferation, differentiation, and apoptosis of immunocompetent cells and characterizes cell cycles associated 
with these processes [6,7]. So, we studied lymphocyte markers such as CD38 + and CD95 +. It is known that $\mathrm{CD} 38+$ is an activation marker represented by a transmembrane glycoprotein, which is considered as a multifunctional protein $[14,16]$. The enzymatic functions of CD38 + provide its main immunoregulatory role; this is the binding of various agents to this receptor, which enhances the synthesis of cytokines, activation of kinases, and protein phosphorylation [6,9]. CD38 + is expressed on immature T- and B-lymphocytes, activated T-lymphocytes, plasmocytes [15]. Analysis of the expression of CD38 + on lymphocytes revealed a significant increase in this marker in groups of patients before treatment. The highest value of CD38 + was observed in groups of patients before treatment with virus carrier. The expression of CD38 + before treatment was $33.2 \pm 1.7 \%$, without virus carrier $-28.4 \pm 1.5 \%$, and normal $-23.4 \pm 0.6 \%$. Therefore, increased expression of CD38 + was observed before the treatment of patients, which is apparently associated with the proliferative activity of specific $\mathrm{T}$ and $\mathrm{B}$ lymphocytes in response to the malignant process and chronic virus carriage. Also, increased expression of CD38 + may be associated with activation of CD3 + CD8 +, CD16 + and CD20 +. According to published data, there is information about the role of APO-1 / Fas (CD95 +) receptors in the process of apoptosis, and its degree is a reflection of the level of lymphocyte apoptosis $[3,6]$. It has been established that an increase in the expression of CD95 + receptors on lymphocytes indicates an excessive and ineffective process of stimulating blood lymphocytes, which indicates an apoptotic pathway for the death of lymphocytes [16]. Binding of CD95 + to the Fas ligand induces apoptosis of cells expressing CD95 +. The analysis showed that in groups of patients increased expression of CD95 + is noted. Moreover, the greatest expression is observed in groups of patients before treatment with virus carrier. Apparently, excessive apoptosis in combination with the activation of the humoral immunity and deep T-cell immunodeficiency contribute to the progression of the disease.

Thus, the analysis of the results allowed us to identify pronounced changes in the cellular component of immunity, which are manifested by a suppression of the expression of CD3 +, CD3 + CD4 +, IRI, increased expression of CD3 + CD8 +, CD16 + and CD20 + cells, as well as increased expression of $\mathrm{CD} 38+, \mathrm{CD} 95+$.

\section{REFERENCES}

1. Ansell S.M., Horwitz S.M., Engert A. et al. Phase L/II study of an anti-CD30-monoclonal antibody (MDX-060) in Hodgkin's lymphoma and anaplastic large-cell lymphoma // J Clin Oncol. 2007. - Vol. 25, N 19. - P. 2764-2769.

2. Atamer T., Artim-Esen- B., Yavus S. et al. Massive post-obstructive disuresis in a patient with Burkitt's lymphoma // Nephrol. Dial. Transplant. 2005. -N 6. - P. 28.

3. Gasses A., Kirby M., Weitzman S. Hepatosplenic gammadelta T-cell lymphoma in a 10-year-old boy successfully treated with hematopoetic sterm cell transplantation // Am. J. Hematol. 2004. - Vol. 12, N 2. - P. 113-114.

4. Hutchison R., Berard C., Shuster J. et al. B-cell lineage confers a favorable outcome among children and adolescents with large-cell lymphoma: a Pediatric Oncology Group study // J. Clin. Oncol. 1995. - Vol. 13. - P. 2023-2032.

5. Jakson R. et al. Extranodal follicular lymphoma: a clinicopathological and genetic analysis of 15 cases arising at non-cutaneous extranodal sites // Histopathology. 2004. - Vol. 44, N 3. - P. 268-276.

6. Kavan P., Kabickova E., Gajdos P. et al. Treatment of pediatric B-cell non-Hodgkin's lymphomas at the Motol Hospital in Prague, Czech Republic: results based on the NHL BFM 90 protocols // Pediatr. Hematol. Oncol. 1999. - Vol. 16, N 3. - P. 201-212.

7. Klein U., Klein G., Ehlin-Henriksson B. et al. Burkitt's lymphoma is a malignancy of mature B-cells expressing somatically mutated V region genes // Mol. Med. 1995. — N 1. - P. 495-506.

8. Kline J., Larson R. Nelarabine in the treatment of refractory T-cell malignant diseases // Expert. Opin. Pharmacother.-2006.-Vol. 7,N 13.-P. 1791-1799.

9. Lindeman N. et al. One patient, two lymphomas. Simultaneous primary gastric marginal zone lymphoma and primary duodenal follicular lymphoma // Arch. Pathol. Lab. Med. -2004. Vol. 128, N9.-P. 1035-1038.

10. Malani A.K., Gupta C., Weigand R.T. et al. Spinal Burkitt's lymphoma in adults // Clin. Lymphoma Myeloma. 2006. - Vol. 6, N 4. - P. 333-336.

11. Manzur A., Zaidi S. Hypopigmented mycosis fungoides in a 10-year-old boy // Dermatol. Online J. 2006. - Vol. 31, N 6. - P. 21.

12. Marte A., Sabatino M., Cautiero P. et al. Unexpected finding of laparoscopic appendectomy: appendix MALT lymphoma in children // Pediatr. Surg. Int. 2007. - Vol. 7. -P. 119-123.

13. Mawanda O.W. Aspects of epidemiological and clinical features of patients with central nervous system Burkitt's lymphoma in Kenia // East. Afr. Med. J. 2004. - Vol. 8. - P. 97103.

14. Mawanda O.W. Clinical characteristics of Burkitt's lymphoma seen in Kenyan patients // East. Afr. Med. J. 2004. - N 8. - P. 78-89.

15. Sharkey R., Karacay H., Johnson C. et al. Pretargeted versus directly targeted radioimmu-notherapy combined with anti-CD20 antybody consolidation therapy of Non-Hodgkin lymphoma//J.Nucl.Med. 2009. - Vol. 17.-P. 123-128.

16. Windsor R., Stiller C., Webb D. Peripheral T-cell lymphoma in childhood: population-based experience in the United Kingdom over 20 years // Pediatr. Blood Cancer. - 2007. -Vol. 11.-P. 87-91. 